\title{
Ensino e Práticas de Promoção da Saúde Durante o Primeiro Ano de Medicina - Unicamp*
}

\author{
Health Promotion Teaching and Practices \\ in the First Year of Medical School at the \\ University of Campinas
}

\author{
Ana Maria Girotti Sperandio \\ Livia Parente Passos ${ }^{l}$ \\ Luiza Manhezi de Freitas Oliveiral \\ Heloísa Sisconeto Bisinotto \\ Ítalo Fernandes do Espírito Santo \\ Carla Cristina Carvalho Celestrino \\ Fernanda Camelo Silval \\ Mayara Satsuki Kunii ${ }^{I}$
}

PALAVRAS-CHAVE

- Educação Médica.

- Promoção da Saúde.

- Gestantes.

- Adolescentes.

KEYWORDS

- Medical Education.

- Health Promotion.

- Pregnant Women.

- Adolescents.

Recebido em: 09/09/2009

Reencaminhdo em: 20/01/2010

Reencaminhdo em: 05/03/2010

Aprovado em: 17/03/2010

REVISTA BRASILEIRA DE EDUCAÇÃO MÉDICA

615

$34(4): 615-621 ; 2010$
* Trabalho apresentado no $12^{\text {th }}$ World Congress on Public Health em abril/maio de 2009 - Istambul/Turquia.

${ }^{1}$ Universidade Estadual de Campinas, Campinas, SP, Brasil. 


\section{INTRODUÇÃO}

Desde a segunda metade do século 20, a área da saúde vem sofrendo transformações constantes devido a avanços nos campos científico e tecnológico. O desenvolvimento científico, sustentado pelas tecnologias de informática e automação, aumentou a eficácia dos procedimentos diagnósticos e terapêuticos, tornando a medicina resolutiva para um grande número de doenças. Dessa forma, a incorporação da tecnologia passou a determinar tanto a organização/gestão de hospitais e clínicas, quanto a formação dos profissionais da área da saúde.

O foco das práticas médicas passou a se concentrar no uso de drogas e de instrumentos de diagnóstico e tratamento. Fundamentos e conceitos da medicina baseada na relação médico-paciente, na escuta, no humanismo e na consideração do paciente como um ser complexo, com dimensões biológica, psíquica e social, foram, muitas vezes, abandonados. O ensino médico concentrou-se nos hospitais e na divisão do trabalho especializado, o que resultou no atual modelo hospitalocêntrico de assistência médica e na departamentalização curricular ${ }^{1}$ (p.137-139).

Apesar de todo esse desenvolvimento da área biomédica, o acesso às novas tecnologias não foi equitativo para toda a população. Nesse contexto, movimentos em defesa da saúde, como o que resultou na Conferência Internacional de Alma-Ata (1978), tiveram importância crucial por promulgar a diretriz "saúde para todos" no ano de 2000, que colocou a saúde como direito dos cidadãos e dever do $\operatorname{Estado}^{2}$ (p.639). A partir de então, abriu-se espaço para maiores discussões sobre a importância da constituição de Sistemas Nacionais de Saúde com ênfase na atenção básica. Em consequência destas mudanças, a formação médica também tem sofrido reformas, tanto do seu conteúdo e método pedagógico, quanto do cenário de práticas.

Na rede básica, espera-se que $80 \%$ das necessidades de saúde da população brasileira sejam atendidas. No Brasil, em 1989, teve início a organização de políticas de saúde com o objetivo de demonstrar a importância de um sistema de saúde público qualificado para atender às necessidades de saúde da população conforme os princípios constitucionais de universalidade, equidade e integralidade das ações estabelecidas para o Sistema Único de Saúde (SUS). Para isso, é fundamental que as instituições de ensino formem profissionais capazes de intervir nessa realidade e de desfrutar deste campo potencial para a prática médica ${ }^{3}$ (p.8-9).

Dessa forma, o governo brasileiro e a Associação Brasileira de Educação Médica (Abem), através do Programa Nacional de Reorientação da Formação Profissional em Saúde (Pró-Saúde) e da substituição do currículo mínimo dos cursos de Medicina pelas Diretrizes Curriculares Nacionais (DCNs), adotaram uma abordagem diferenciada para o ensino médico com o objetivo de reorientar a formação profissional. O objetivo central do Pró-Saúde é a integração ensino-serviço, de modo a assegurar uma abordagem integral do processo saúde-doença com ênfase na atenção básica, e, assim, transformar o processo de ensino e prestação de serviços à população. Para isso, é preciso que as instituições de ensino desloquem o atual eixo da formação - centrado na assistência individual prestada em unidades especializadas - para um processo voltado às necessidades sociais, econômicas e culturais da população ${ }^{4}$ (p.12-14). As Diretrizes Curriculares Nacionais apontam a necessidade de formar médicos capazes de integrar as dimensões biológica, psicológica, social e ambiental no processo saúde-doença; de atuar em equipes multiprofissionais para a produção de saúde e que tenham formação geral, humanista, crítica e reflexiva. Logo, o conteúdo curricular deve estar pautado em metodologias que incentivem a participação ativa do estudante na construção do conhecimento, vinculando a formação médico-acadêmica às necessidades sociais da saúde com base nos princípios do SUS. Essa integração entre ensino, pesquisa e extensão/assistência propicia a interação precoce dos alunos com os usuários e profissionais do Sistema de Saúde, a qual permite ao estudante lidar com problemas reais e assumir responsabilidades crescentes como agente prestador de cuidados e atenção ${ }^{5}$ (p.77).

Com essa linha de pensamento, em 2001, a Faculdade de Ciências Médicas da Universidade Estadual de Campinas (FCM-Unicamp) adotou as Novas Diretrizes Curriculares para os cursos da área de saúde. Como metodologia para a implantação dessas novas diretrizes, a FCM-Unicamp reformulou o currículo do seu curso de Medicina com a criação, mais especificamente, das disciplinas Ações de Saúde Pública, Saúde e Sociedade, e Atenção Integral à Saúde, ministradas, respectivamente, no primeiro, segundo e quarto anos.

Este trabalho tem por eixo a disciplina obrigatória Ações de Saúde Pública, cuja carga horária de 120 horas foi ministrada durante o ano de 2008 aos alunos do primeiro ano do curso de Medicina da FCM-Unicamp. O objetivo geral deste módulo é introduzir os temas Saúde Coletiva e os conceitos de política pública na área da saúde, o Sistema Único de Saúde e o caráter biopsicossocial do processo saúde-doença e do adoecer no âmbito individual e coletivo. Além disso, o módulo tem como objetivos específicos:

- Contribuir para a compreensão da produção de saúde como um processo que está além do exclusivamente médico ou biológico; 
- Analisar os principais determinantes do processo de saúde/doença com ênfase no território e nas desigualdades;

- Colocar os alunos em contato com a rede básica do SUS, analisando a atenção à saúde e a sua relação com o processo de produção da saúde;

- Propiciar a aquisição de competência para um relacionamento ético e humanizado com equipes, usuários e comunidades, através da realização de ações simples junto aos serviços territoriais de saúde ${ }^{6}$. (p. 43)

Aproximando-se os objetivos da disciplina e os princípios da promoção da saúde - a ampliação da autonomia das pessoas de determinado território, a participação social, a intersetorialidade, a construção ou resgate de políticas públicas saudáveis e sua sustentabilidade - , criam-se possibilidades de exercício teórico e prático para desenvolver projetos de intervenção no campo nessa perspectiva, com ação focal e trabalho na formação em saúde.

Segundo Labonte ${ }^{7}$, a promoção da saúde não se restringe a conhecer os motivos das iniquidades, que são injustas e mutáveis, mas deve englobar políticas públicas para reduzi-las. $\mathrm{O}$ autor acredita que para o planejamento e gestão deve-se considerar a decisão de onde intervir e sugere que os promotores de saúde aprendam sobre o desenvolvimento econômico e do território (p. 195). Já para Sperandio ${ }^{8}$, a promoção da saúde desempenha um papel fundamental no desenvolvimento e aplicação das políticas públicas saudáveis integradas e transversais, na busca da produção de saúde, e possibilita a interação das diferentes áreas do saber (p.12).

Segundo a perspectiva da disciplina Ações de Saúde Pública, a análise do território tem papel fundamental para o conhecimento da população local. O território é entendido como o resultado das relações humanas dentro do espaço físico. Dessa forma, os componentes social, econômico, cultural e ambiental que envolvem a população determinam as características e particularidades de um território específico. Essa integração promovida pelo território faz com que o espaço físico esteja intimamente relacionado com o processo saúde-doença. Por isso, o conhecimento do território, da realidade e necessidades dos moradores através do contato direto com a população local é fundamental para o desenvolvimento e sucesso de qualquer interferência ${ }^{9}$ (p.321-30). A análise do território demonstra, muitas vezes, realidades distintas e conflitantes num mesmo espaço. Isto significa que os projetos de interferência devem estar atrelados à adoção de políticas específicas para o território, visando à promoção da saúde ${ }^{10}$ (p.64-6).
Através da observação da área de abrangência do Centro de Saúde (CS) São Quirino, no município de Campinas (SP), e do contato com a população local, os alunos da XLVI Turma de Medicina da FCM-Unicamp constataram a presença de grande número de adolescentes grávidas no território. Conforme orientação do módulo de Ações de Saúde Pública, os alunos deveriam elaborar um Projeto de Intervenção no CS, de maneira participativa com a população da comunidade e da equipe profissional do CS.

A gestação na adolescência tem sido considerada um problema de saúde pública em virtude de seu aumento no final do século passado e de suas repercussões significativas tanto para o indivíduo quanto para a sociedade em geral ${ }^{11}$ (p.480), ${ }^{12}$ (p.185). Para a adolescente, a gravidez precoce dificulta o desenvolvimento pessoal, pois acarreta perda da liberdade, comprometimento dos projetos de estudo e limitação das perspectivas de ingresso no mercado de trabalho e de ascensão social ${ }^{11}$ (p.481), ${ }^{12}$ (p.184). Em 2000, a média de idade das adolescentes gestantes no município de Campinas foi de 17,2 anos, o que coincide com a idade $\operatorname{escolar}^{14}$ (p.25).

A maternidade e a constituição da família afetam a escolarização das mães adolescentes, pois estas passam a assumir papéis da vida adulta que, muitas vezes, são incompatíveis com os estudos ${ }^{13}$ (p.300). Como reflexo da gravidez precoce, o baixo nível educacional tem impacto negativo, também, no potencial de ascensão econômica das comunidades e de todo o País ${ }^{12}$ (p.184). A gravidez na adolescência está associada, ain$\mathrm{da}$, a maior frequência de prematuridade, baixo peso ao nascer e maior risco de morte da criança no primeiro ano de vida ${ }^{12}$ (p.184-185), ${ }^{13}$ (p.292).

A chamada epidemia da maternidade na adolescência foi reconhecida por volta de 1970, quando as taxas de fecundidade já começavam a cair em países do Primeiro Mundo. Contudo, estatísticas mostram que, nos últimos 30 anos, a taxa de fecundidade em adolescentes aumentou no Brasil, apesar da queda ocorrida na faixa etária adulta. Com relação ao total de partos realizados em hospitais conveniados ao SUS, a participação da faixa etária dos 10 aos 19 anos passou de 22,34\% em 1993 para 26,5\% em $1997^{12}$ (p. 177-178). A Pesquisa Nacional sobre Demografia e Saúde, realizada em 1996 no Brasil, apontou que $18 \%$ das mulheres entre 15 e 19 anos já haviam iniciado a vida sexual, sendo que deste total 14,3\% referiram pelo menos uma gravidez ${ }^{13}$ (p. 292).

Na literatura, algumas variáveis são consideradas fatores de risco potenciais para a gravidez na adolescência. Entre esses fatores destacam-se o início precoce da vida sexual, que resulta em maior tempo de exposição à concepção, níveis escolar e socioeconômico baixos, baixa qualidade da informação sobre mé- 
todos contraceptivos e desconhecimento da fisiologia reprodutiva, tal como a identificação do período fértil ${ }^{11}$ (p. 480-481).

Por toda a relevância individual e coletiva do tema da gravidez na adolescência, os alunos da XLVI Turma de Medicina da FCM-Unicamp envolvidos no projeto organizaram um grupo de gestantes no CS São Quirino. Este foi aceito pela coordenação do CS, destacando a necessidade de trabalhar com essa população.

\section{OBJETIVO GERAL}

Colaborar com a melhoria da qualidade de vida das adolescentes grávidas e de seus filhos, com o desenvolvimento e a implantação de estratégias de promoção da saúde no Centro de Saúde São Quirino, em Campinas (SP).

\section{OBJETIVOS ESPECÍFICOS}

- Envolver a equipe profissional do Centro de Saúde São Quirino, em Campinas (SP), na elaboração e realização do projeto, para garantir sua sustentabilidade;

- Organizar um grupo de acompanhamento de gestantes no CS que reforce a importância da atuação da tríade universidade, serviço de saúde e comunidade;

- Sensibilizar, capacitar e mobilizar os alunos do primeiro ano de Medicina para o aprendizado e a prática de ações de promoção da saúde, valorizando a importância dos processos desenvolvidos para a formação médica.

\section{METODOLOGIA DE TRABALHO}

No início de 2008, os 110 alunos do primeiro ano do curso de Medicina foram distribuídos entre oito Centros de Saúde de Campinas para realizar trabalhos segundo as necessidades específicas das regiões às quais cada CS pertencia. O CS São Quirino recebeu 14 desses alunos, que visitaram o território, conversaram com as agentes comunitárias e a população local, e conheceram a realidade da região. Em reunião após as visitas, os alunos relataram uma grande quantidade de adolescentes gestantes a transitar pelas ruas do bairro ou em atendimento na unidade. Como o grupo deveria determinar uma população específica para a construção de um projeto de intervenção, essa parcela foi a escolhida. Dessa forma, o grupo elaborou um projeto de intervenção que serviria aos requisitos da disciplina e trabalharia de forma direta com essa parcela da população, propondo desenvolver ações na perspectiva da promoção da saúde.

Para verificar a viabilidade dessa amostra, seria necessário um levantamento prévio nos arquivos da unidade. Esse estudo foi realizado para determinar o número de adolescentes grávidas assistidas pelo CS, seus endereços e idade gestacional, através de levantamentos no livro de gestantes e nos prontuários. Decidiu-se analisar os dados dos meses de maio a agosto, pois interessava ao grupo a participação da adolescente com idade gestacional de um a seis meses na data da primeira oficina. Esse critério foi utilizado para que a gestante pudesse relatar as sensações e experiências que estava tendo durante a gravidez e para que seu deslocamento até o CS e sua permanência nele não lhe fossem incômodos. Essa análise revelou um total 57 de gestantes entre os meses de maio e agosto de 2008, sendo que 17 destas eram adolescentes.

Após a identificação e mapeamento das gestantes e escolha do projeto de intervenção, fez-se um levantamento das necessidades de saber das grávidas por meio de um questionário elaborado pela equipe com o objetivo de sistematizar as necessidades de conhecimento das gestantes com relação à gravidez. Para a entrevista, foram selecionadas gestantes com idades entre 12 e 21 anos, com tempo gestacional entre um e seis meses na data da primeira oficina, que realizavam acompanhamento pré-natal no CS São Quirino, em Campinas (SP), e que residiam na área de abrangência da unidade. Com a ajuda do mapa da área de abrangência do CS, as gestantes foram localizadas, e as visitas foram organizadas junto às agentes comunitárias para a aplicação do questionário.

Após a interpretação dos dados obtidos no levantamento, a equipe discutiu quais seriam as necessidades de saber das gestantes, para construir um modelo de trabalho que abordasse da maneira mais didática possível os assuntos desconhecidos por elas, otimizando os recursos humanos no CS São Quirino e fazendo com que as próprias gestantes selecionassem e decidissem quais seriam os conteúdos discutidos nos encontros. O trabalho foi coordenado e acompanhado pela professora responsável e realizado pelos alunos do grupo e profissionais do Centro de Saúde.

Decidiu-se que o grupo de acompanhamento seria composto por encontros semanais, nos quais as dúvidas das gestantes seriam sanadas utilizando-se diversas formas de abordagem, como rodas de conversa, folders, oficinas, teatro, etc.

No logotipo, pode-se ver a clara associação entre gravidez e vida, e entre mãe e filho, o que era uma das ideias do grupo. Ao aproveitar o foco da palavra "vida" para a própria existência do ser humano, três frentes de trabalho foram elaboradas e desenvolvidas:

- VIDA 1 - a mãe e a gravidez: essa fase contou com discussões sobre medos e inseguranças durante a gestação, cuidados com o corpo, hora do parto e visita à maternidade;

— VIDA 2 - o bebê: vacinação, higiene e amamentação e seus benefícios;

— 2 VIDAS - a mãe e o bebê juntos: seus direitos — atendimentos prioritários e direitos trabalhistas. 


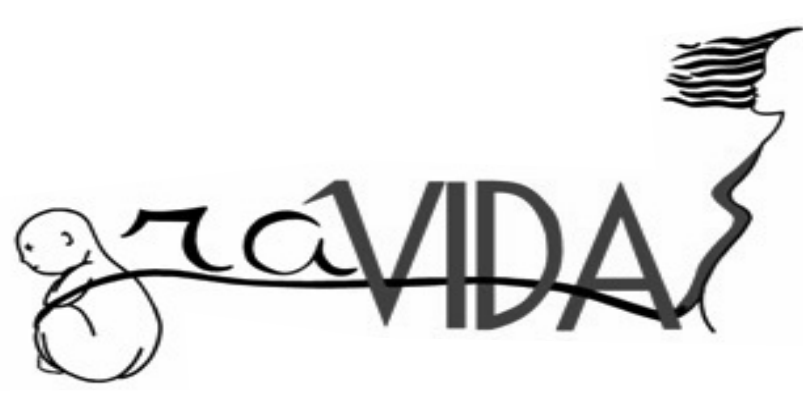

Essa proposta de trabalho reforça inclusive os princípios da promoção da saúde, principalmente os da autonomia, intersetorialidade e participação social.

Para as reuniões, os alunos convidaram funcionários da unidade de saúde e elaboraram folders, cartazes, apostilas, etc. Durante todo o projeto, elaborou-se um diário de bordo que registrava todas as atividades do dia, para leitura e acompanhamento. Além disso, foi criado o álbum "Meu Caderninho", onde se registravam sensações e fotos das gestantes. As atividades foram conduzidas com o apoio e a participação da equipe do CS através de um trabalho de promoção da saúde com ações educativas, visando à continuidade do grupo em outros anos.

\section{RESULTADOS E DISCUSSÃO}

Todas as gestantes selecionadas foram visitadas, embora nem todas estivessem em casa no momento das entrevistas, e convidadas a responder ao questionário e a comparecer ao CS nos dias indicados.

De modo geral, o trabalho de grupo atingiu sua meta, pois a equipe do CS demonstrou interesse em continuar o trabalho com as adolescentes, e os profissionais se envolveram tanto na elaboração quanto na execução do projeto, o que assegurou melhorias e a sustentabilidade do grupo. Além disso, outro resultado desse interesse foi a incorporação do grupo de gestantes às atividades rotineiras do CS a partir do ano de 2009.

Em relação às gestantes, o grupo encontrou algumas dificuldades devido à baixa adesão. Das 15 gestantes selecionadas, cinco não foram entrevistadas por mudança de endereço não informada ao CS ou por ausência em suas residências no momento da entrevista. Nove das dez gestantes que foram entrevistadas e convidadas a comparecer às reuniões participaram destas, mas a frequência foi irregular. As agentes de saúde apontaram possíveis motivos para essa irregularidade: as grandes distâncias entre as residências das gestantes e o CS, temperaturas elevadas, próprias da região, além da indisponibilidade de horário das adolescentes para comparecerem aos encontros do grupo. De acordo com Dall'Agnese e Geib ${ }^{15}$, a menor adesão das gestantes ao programa de assistência pré-natal deve-se a fatores como o preconceito para com o serviço público e o desinteresse das usuárias ${ }^{15}$ (p. 16). Contudo, as causas reais dessa pequena adesão ainda devem ser mais bem avaliadas.

Para os alunos de Medicina, a formação em promoção da saúde e a realização do projeto de intervenção proporcionaram um contato amplo com a dinâmica de um CS, com as principais estratégias de promoção da saúde e com a política do SUS. Essas experiências lhes permitiram observar a importância da inserção do trabalho em promoção da saúde nas atividades diárias de uma unidade, bem como as repercussões e resultados para os usuários do serviço.

Foram realizadas avaliações orais a cada encontro tanto das grávidas como dos alunos, com o objetivo de trabalhar as dificuldades. Ao final do projeto, uma avaliação geral com a aplicação de um questionário permitiu constatar diferentes pontos:

- Necessidade de saber das gestantes sobre assuntos abordados no grupo;

- Interesse no projeto tanto das gestantes quanto dos profissionais do CS;

- As oficinas forneceram informações de acordo com as necessidades de conhecimento básico por parte das adolescentes;

— Importância do processo de educação em saúde;

- Adequação do conteúdo e da dinâmica utilizados às necessidades de conhecimento das gestantes;

- Relevância da construção de um clima de intimidade para as gestantes sanarem suas dúvidas, em razão do ambiente informal e coletivo;

- Desejo da equipe do CS de continuar com o grupo, já que se trata de uma estratégia de promoção da saúde que transmite às gestantes aspectos fundamentais para compartilhar conhecimentos e aproximar mãe, filho e CS, com resultados positivos ao longo da gestação e após o nascimento;

— Viabilidade de operacionalização, dentro da rotina do CS, de um grupo de gestantes que inclua profissionais das mais diversas áreas.

Essa avaliação reafirma a necessidade de elaborar projetos de intervenção que contemplem as necessidades das usuárias gestantes adolescentes e do serviço de saúde pública, transversalizando a atuação da universidade.

\section{CONCLUSÃO}

Em consonância com as novas Diretrizes Curriculares para os cursos da área de saúde, os alunos tomaram conhecimento das 
necessidades de um território e dos desafios e limitações que envolvem os trabalhos de promoção da saúde e prevenção de doenças na Atenção Básica. Além disso, tiveram a oportunidade ímpar de conhecer, em um momento inicial do curso médico, a dinâmica de um Centro de Saúde.

O desenvolvimento de um projeto de intervenção na perspectiva da promoção da saúde junto à população e ao Centro de Saúde foi de extrema relevância para o aprendizado e experiência dos alunos, com influência impactante em sua formação profissional. A comunicação com as adolescentes também trouxe um aprendizado aos alunos, que, algumas vezes, tiveram que repensar o projeto de acordo com as demandas das gestantes, que nem sempre haviam sido previstas pela equipe.

O trabalho numa equipe multidisciplinar com os profissionais do CS revelou ao grupo a importância de abordar o sujeito de forma integral, agregando os conhecimentos das diversas áreas ao se trabalhar com um processo biológico. A partir do contato direto com a população usuária do CS, os estudantes tiveram a oportunidade de analisar não apenas os componentes biológicos do processo saúde-doença, mas também os determinantes sociais que influenciam a geração de problemas graves e frequentes na saúde pública brasileira, como os envolvidos no estudo.

Com relação aos profissionais do CS, ficou evidente que o projeto dos alunos do primeiro ano de Medicina serviu como incentivo necessário à formação de outros grupos de gestantes, o que já era uma demanda da população local a ser trabalhada. A equipe se mostrou bastante interessada e engajada em continuar e ampliar o trabalho desenvolvido, para manter um grupo permanente de acompanhamento de gestantes.

Para as gestantes, o projeto foi importante por melhorar sua qualidade de vida, assim como a de seus bebês. Ao esclarecer as dúvidas das gestantes, o grupo atuou também na transformação da percepção de cada uma sobre os processos e as mudanças envolvidos no período da gestação. Tal transformação resultou na melhoria da relação entre a gestante e seu bebê, e na aproximação entre a gestante e o CS. Esta aproximação do CS, junto à nova perspectiva da formação do profissional da saúde, propicia uma nova forma de pensar saúde, desencadeando processos de aprendizado na população, nos alunos e no profissional do Centro de Saúde na perspectiva da promoção da saúde.

\section{AGRADECIMENTOS}

Agradecemos à Equipe do CS São Quirino pela colaboração na execução do projeto; à Prof ${ }^{\underline{a}}$ Dra $^{\underline{a}}$ Rosana Onocko Campos e ao Prof Dr Gastão Wagner Campos pela viabilização e organização do projeto.

\section{REFERÊNCIAS}

1. Amoretti R. Educação Médica Diante das Necessidades Sociais em Saúde. Rev Bras Educ Med. 2005;29(2) \;136-46

2. Westphal MF. Promoção da saúde e prevenção de doenças. In: Campos et al. orgs. Tratado de saúde coletiva. Rio de Janeiro: Fiocruz; 2006. p.635-62

3. Campos GWS. Papel da Rede de Atenção Básica em Saúde na Formação Médica: diretrizes. Cad ABEM. 2007;3:p.6-10

4. Brasil. Ministério da Saúde e Ministério da Educação. Programa Nacional de Reorientação da Formação Profissional em Saúde - Pró-Saúde: objetivos, implementação e desenvolvimento potencial. Brasília: MS; 2007. p.7-26. (Série C. Projetos, Programas e Relatórios)

5. Stella RCR, Campos JJB. Histórico da Construção das Diretrizes Curriculares Nacionais na Graduação em Medicina no Brasil. Cad ABEM; 2006;2:p.77

6. Universidade Estadual de Campinas. MD 142 - Ações de Saúde Pública I. Programa dos módulos de ensino do curso de medicina: primeiro ano $-1^{\mathrm{o}}$ semestre. Campinas: Unicamp;2008. p.43-51

7. Labonte R. Globalization and health promotion: the evidence challenge. In: McQueen DV, Jones CM. Global perspectives on health promotion effectiveness. Nova Iorque: Springer Science; 2007. p.181-200

8. Sperandio AMG, Bueno RN, Correa CR, Matida A, Spadacio $C$, Bodstein $R$, et al. O Processo de avaliação das experiências locais do projeto de ações intersetorias em promoção da saúde - AIPS: buscando as igualdades a partir das diferenças. Rio de Janeiro: Abrasco; 2009

9. Santos M. O lugar e o cotidiano. In: A natureza do espaço. São Paulo: Edusp; 2002. Cap. 14, p. 313-39

10. Sousa MAA. Uso do território e saúde: refletindo sobre "municípios saudáveis". In: Sperandio, AMG, org. O processo de construção da rede de municípios potencialmente saudáveis. Campinas: IPES; 2004. v.2. p.57-77

11. Silva JLCP, Belo MAV. Conhecimento, Atitude e Prática Sobre Métodos Anticoncepcionais Entre Gestantes Adolescentes. Rev Saúde Pública. 2004;38(4):479-87

12. Chalem E, Mitsuhiro SS, Ferri CP, Barros MCM, Guinsburg R, Laranjeira R. Gravidez na Adolescência: Perfil Sócio-demográfico e Comportamental de uma População na Periferia de São Paulo. Cad Saúde Pública. 2007;23(1):177-86

13. Figueiró AC. Condições de Vida e Saúde Reprodutiva de Adolescentes Residentes na Comunidade de Roda de Fogo, Recife. Rev Bras Saúde Mater Infant. 2002;2(3):291-302

14. Batista RFL. Condições de vida e saúde de gestantes adolescentes residentes no município de Campinas. Campinas; 2001. Mestrado [Dissertação] - Faculdade de Ciências Médicas, Universidade Estadual de Campinas; 
15. Dall'Agnese LE, Geib LTC. Absenteísmo ao Programa de Assistência Pré-Natal: Motivos Alegados por Mães de Crianças Prematuras. Bol Saúde. 2003;17(1):9-20

\section{CONTRIBUIÇÃO DOS AUTORES}

Ana Maria Girotti Sperandio contribuiu para a concepção e desenho do estudo, aquisição de dados, análise e interpretação de dados; supervisão, elaboração do artigo, elaboração de versão inicial do artigo ou sua revisão crítica para conteúdo intelectual significativo. Lívia Parente Passos contribuiu na elaboraão e correções: resumo, introdução, objetivos, metodologia de trabalho, resultados e discussão, conclusão, organização das referencias bibliográficas e do artigo. Luiza Manhezi de Freitas Oliveira contribuiu na elaboração e correções: resumo, introdução, objetivos, metodologia de trabalho, resultados e discussão, conclusão. Heloísa Sisconeto Bisinotto contribuiu na elaboração da introdução, objetivos e organização das referências bibliográficas. Ítalo Fernandes do Espírito Santo contribuiu na elaboração da introdução e da Metodologia de trabalho. Carla Cristina Carvalho Celestrino contribuiu na metodologia e resultados. Fernanda Camelo Silva colaborou na metodologia, resultados e considerações. Mayara Stsuki Kunii colaborou na elaboração dos resultados e das considerações.

\section{CONFLITO DE INTERESSES}

Declarou não haver.

\section{ENDEREÇO PARA CORRESPONDÊNCIA}

Ana Maria G. Sperandio

Av. Alaor Maria de Barros, 1050 — Ed. Paratí, 81

Alphaville - Campinas

CEP. 13098-393 SP

E-mail: amgspera@uol.com.br 